\title{
Spin injection and transport in single-crystalline organic spin valves based on TIPS-pentacene
}

\author{
Ying Wang ${ }^{1}$, Jiarong Yao ${ }^{1}$, Shuaishuai Ding ${ }^{1 *}$, Siyu Guo ${ }^{1}$, Dapeng Cui ${ }^{2}$, Xinyue Wang ${ }^{3}$, \\ Shuyuan Yang ${ }^{1}$, Lijuan Zhang ${ }^{1}$, Xinzi Tian ${ }^{1}, \mathrm{Di}_{\mathrm{Wu}}{ }^{2}$, Chao Jin ${ }^{3}$, Rongjin $\mathrm{Li}^{{ }^{*}}$ and Wenping $\mathrm{Hu}^{1,4}$
}

\begin{abstract}
Single crystals of organic semiconductors with perfect crystal structure and minimal density of defects can exhibit high mobility and low spin scattering compared with their amorphous or polycrystalline counterparts. Therefore, these materials are promising candidates as the spin transport media to obtain long spin relaxation times and spin diffusion lengths in spintronic devices. However, the investigation of spin injection and transport properties in organic single crystals is hindered by the inability to construct devices such as single-crystalline organic spin valves (OSVs). Herein, thin and large organic single crystals of 6,13-bis(triisopropylsilylethynyl)pentacene (TIPS-pentacene) were grown on a liquid substrate and transferred to a target substrate carrying ferromagnetic electrodes to construct single-crystalline OSVs. The magnetoresistance (MR) responses of the single crystals were investigated to study their spin injection and transport properties. MR value as high as $17 \%$ was probed with an intermediate layer thickness of $269 \mathrm{~nm}$. More importantly, spin transport was still observed in a single crystal of a thickness up to $457 \mathrm{~nm}$, which was much larger than that of polycrystalline thin film. Our research provides a general methodology for constructing single-crystalline OSVs and paves the way to probe the intrinsic spin transport properties of organic semiconductors based on single crystals.
\end{abstract}

Keywords: organic single crystal, organic semiconductor, magnetoresistance, organic spin valve, spintronics

\section{INTRODUCTION}

Spintronics considers not only the charge of electrons but also their spin degree of freedom in electronic devices [1-4]. Spintronics shows great potential in applications such as information storage and processing [2]. In particular, organic semiconductors have aroused considerable research attention because they are a promising medium for spin transport due to their long spin relaxation time [3,5-8]. Organic semiconductors are mainly composed of light elements such as carbon and hydrogen with weak spin-orbit coupling (SOC) interactions [3,9-11], which is the primary reason for spin-flipping in most materials [12-14]. Another source of spin-flipping is hyperfine interaction (HFI), which is mainly caused by hydrogen atoms and is only strong in localized carriers [15]. As a result, delocalized carriers in organic semiconductors are expected to have long spin relaxation times $[9,10,12]$. In addition, organic materials have desirable properties, such as solution processability, mechanical flexibility, and chemical tunability, making them promising alternatives in spintronic applications compared with conventional inorganic materials $[16,17]$.

Vertical organic spin valves (OSVs), which are composed of two different ferromagnetic (FM) electrodes separated by organic semiconductors, are a type of primitive spintronic device to investigate spin-polarized charge injection and transport in organic semiconductors [1,3,10,18-20]. In 2004, Xiong et al. [21] reported the first vertical OSV using tris(8-hydroxyquinoline)aluminum

\footnotetext{
${ }^{1}$ Tianjin Key Laboratory of Molecular Optoelectronic Sciences, Department of Chemistry, School of Science, Tianjin University and Collaborative Innovation Center of Chemical Science and Engineering, Tianjin 300072, China

${ }^{2}$ National Laboratory of Solid State Microstructures, Department of Materials Science and Engineering and Jiangsu Key Laboratory for Artificial Functional Materials, Nanjing University, Nanjing 210093, China

${ }^{3}$ Tianjin Key Laboratory of Low Dimensional Materials Physics and Preparing Technology, Department of Physics, School of Science, Tianjin University, Tianjin 300072, China

${ }^{4}$ Joint School of National University of Singapore and Tianjin University, International Campus of Tianjin University, Binhai New City, Fuzhou 350207, China

*Corresponding authors (emails: dingshuaishuai@tju.edu.cn (Ding S); lirj@tju.edu.cn (Li R))
} 
$\left(\mathrm{Alq}_{3}\right)$ as the organic intermediate layer and observed a negative magnetoresistance (MR) of $40 \%$ at $T=11 \mathrm{~K}$ with an intermediate layer thickness of $130 \mathrm{~nm}$. Subsequently, a variety of organic semiconductors including small molecules (e.g., fullerene [5,6,22,23] and pentacene [24]) and $\pi$-conjugated polymers (e.g., poly(dioctyloxy)phenylenevinylene (DOOPPV) [25,26], and poly(3-hexylthiophene-2,5-diyl)regioregular (RR-P3HT) [27,28]) were studied extensively and MR effect was observed. However, all of the above organic intermediate layers in the OSVs are either amorphous or polycrystalline structures, in which the high density of defects and grain boundaries act as spin-flipping centers and reduce their spin diffusion length, greatly limiting their practical application in organic spintronic devices [3,5,29].

In contrast to amorphous or polycrystalline organic solids, organic single crystals show long-range order with minimal density of defects, which enables the investigation of the intrinsic spin-polarized carrier transport properties of organic materials [30-32]. Moreover, they might be a class of promising candidates for obtaining long spin relaxation times and spin diffusion lengths $[3,30]$. In recent years, many attempts have been made to fabricate spintronic devices based on organic single crystals. For example, in 2010, Naber et al. [33] reported the construction of horizontal spin-valve field-effect transistors (FETs) based on rubrene single crystals with Co-FM electrodes. By taking advantage of the high carrier mobility and low density of charge and spin scattering centers in rubrene single crystals, they expected the observation of a long spin diffusion length and the realization of OSV effect. Unfortunately, the expected OSV effect was not observed, possibly due to the very long $(60 \mu \mathrm{m})$ channel length in the spin-valve FETs. Ding et al. [34] reported the organic MR (OMAR) of organic single crystals with high magnetic-field sensitivity. In 2017, Tsurumi et al. [14] estimated that the spin diffusion length could be as long as $840 \mathrm{~nm}$ at $300 \mathrm{~K}$ and surprisingly $1.6 \mu \mathrm{m}$ at $50 \mathrm{~K}$ in a single crystal of 3,11-didecyldinaphtho[2,3-d:2',3'- $\left.d^{\prime}\right]$ benzo[1,2-b:4,5- $\left.b^{\prime}\right]$ dithiophene $\left(\mathrm{C}_{10}\right.$-DNBDT-NW) according to the Einstein relationship, indicating the great potential of organic single crystals as intermediate materials for spintronic applications. However, due to the challenge of growing thin and large-area organic single crystals and the inability to transfer such crystals to FM electrodes for device fabrication, working vertical OSVs based on organic single crystals have not yet been reported.

Recently, we have developed a strategy to grow thin and large-area organic single crystals with different thick- nesses on glycerol surface [35]. These semi-freestanding crystals on liquid surfaces can be transferred to arbitrary substrates to construct various devices. Herein, OSVs based on organic single crystals of 6,13-bis(triisopropylsilylethynyl)pentacene (TIPS-pentacene) were constructed and their spin injection and transport properties were investigated in comparison with those based on polycrystalline thin films. TIPS-pentacene was used as the architype organic semiconductor not only because of its good solubility in common organic solvents, but also its high mobility, especially in arrays of aligned single crystals [36-40]. In the single-crystalline OSVs, MR up to $17 \%$ was recorded at a low temperature. In particular, the MR signal was detectable with an intermediate layer as thick as $457 \mathrm{~nm}$, which was much longer than that of polycrystalline thin films. As far as we know, this is the first working vertical OSV based on organic single crystals. Our study provides a reliable method to construct OSVs based on organic single crystals to explore their intrinsic spin transport properties and provides a framework for achieving long spin diffusion lengths for practical applications of organic spintronic devices.

\section{EXPERIMENTAL SECTION}

\section{Materials}

TIPS-pentacene powders were purchased from Aiwang Chemical Technology Co., Ltd. and used without purification. $\mathrm{La}_{2 / 3} \mathrm{Sr}_{1 / 3} \mathrm{MnO}_{3}$ (LSMO) films with a thickness of $70 \mathrm{~nm}$ were deposited onto $\mathrm{SrTiO}_{3}$ (STO) substrates at $700^{\circ} \mathrm{C}$ with radio frequency magnetron sputtering technique. The ratio of $\mathrm{Ar}$ and $\mathrm{O}_{2}$ flux was 1:1, and the sputtering pressure was $0.5 \mathrm{~Pa}$. The films were subsequently annealed at $700^{\circ} \mathrm{C}$ for $1 \mathrm{~h}$ in a muffle furnace under pure $\mathrm{O}_{2}$ at $260 \mathrm{~Pa}$, followed by slow cooling to room temperature at $5 \mathrm{~K} \mathrm{~min}^{-1}$. Then, the as-prepared LSMO films were cleaned with acetone and isopropanol before use. The LSMO substrates were reused multiple times without noticeable degradation.

\section{Sample preparation}

TIPS-pentacene powders were dissolved in toluene and sonicated for $10 \mathrm{~min}$ to obtain solutions with concentrations ranging from 1 to $2.5 \mathrm{mg} \mathrm{mL}^{-1}$. Glycerol $(35 \mathrm{~mL})$ was added into a weighing bottle (a cylinder container with a diameter of $70 \mathrm{~mm}$ and a height of $40 \mathrm{~mm}$ ) as a liquid substrate for crystal growth. A fixed volume of $100 \mu \mathrm{L}$ of the as-prepared solution was added dropwise onto the surface of the glycerol and dried at a low temperature $\left(10^{\circ} \mathrm{C}\right)$ for $24 \mathrm{~h}$ to grow single crystals. 
The as-grown single crystals floating on the surface of the liquid substrate were then transferred to the LSMO/STO substrate. Polycrystalline thin films were thermally evaporated on top of the LSMO/STO substrate at a rate of $0.1 \AA \mathrm{s}^{-1}$ and a base pressure of $1 \times 10^{-6}$ Torr $(1$ Torr $=$ $1.33322 \times 10^{2} \mathrm{~Pa}$ ). Top Au/Co electrodes were fabricated by vacuum thermal evaporation. Co electrodes $(10 \mathrm{~nm})$ were thermally evaporated onto the TIPS-pentacene crystals at a rate of $0.07 \AA \mathrm{s}^{-1}$ through a shadow mask at a base pressure of $1 \times 10^{-6}$ Torr. Au films $(60 \mathrm{~nm})$ were deposited on top of the Co electrodes at a rate of around $0.1 \AA \mathrm{s}^{-1}$ as a capping layer to prevent the oxidation of Co films. The junction area was $200 \mu \mathrm{m} \times 200 \mu \mathrm{m}$.

\section{Characterizations}

The morphologies and thicknesses of the TIPS-pentacene single crystals and polycrystalline thin films were characterized by tapping-mode atomic force microscopy (AFM, Bruker Dimension Icon). Typical optical microscopy (OM) and polarized OM (POM) images were carried out on a Nikon ECLIPSE Ci-POL. Bright-field transmission electron microscopy (TEM) and selected area electron diffraction (SAED) measurements were performed using an FEI Talos F200X G2. X-ray diffraction (XRD) measurements were performed using a Rigaku Smartlab diffractometer with monochromatic $\mathrm{Cu}$ $\mathrm{K} \alpha(\lambda=1.541 \AA)$ radiation. The charge transport properties of the TIPS-pentacene single crystals and polycrystalline thin films were characterized using a Keithley 4200 SCS connected to a Micromanipulator 6150 probe station in ambient air at room temperature. The electrical and magnetic properties were measured using a Quantum Design physical property measurement system (PPMS DynaCool). The MR curves were measured using a standard four-probe technique between 30 and $100 \mathrm{~K}$ under an external in-plane magnetic field. The hysteresis loop measurements of the two FM electrodes were performed using the vibrating sample magnetometer of the PPMS. The current $(I)$-voltage $(V)$ measurements of devices were performed using a Keithley 2636B sourcemeasure unit.

\section{RESULTS AND DISCUSSION}

\section{Crystal growth and characterization}

Glycerol was chosen as a liquid substrate. The high surface tension together with the high viscosity of glycerol can promote the spreading and fix the position of the solution during evaporation, which was favorable for the production of thin and large-area single crystals [35]. TIPS-pentacene, a high-mobility material for organic FETs (OFETs), was chosen as the architype organic semiconductor. The as-prepared solution $(100 \mu \mathrm{L})$ was dropped onto the surface of the glycerol. After the complete evaporation of the solvent, large-area and thin crystals were grown on the liquid surface. The as-grown crystals were transferred to the target substrates by placing the substrates on the surface of the crystals upside down and pulling out of the liquid surface (Fig. 1a-c). TIPS-pentacene crystals transferred to the target sub-

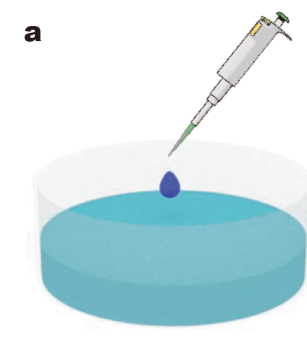

b

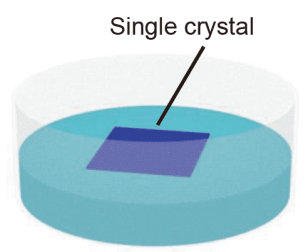

e

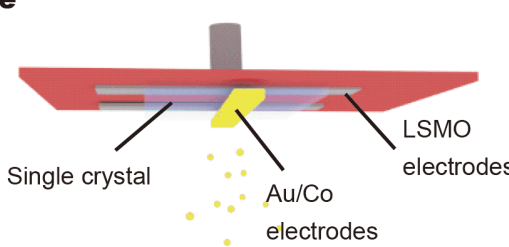

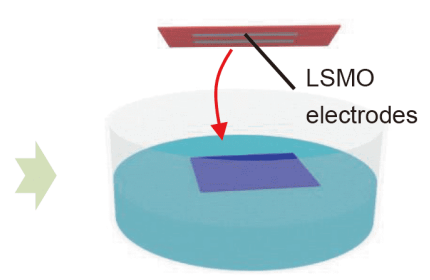

d

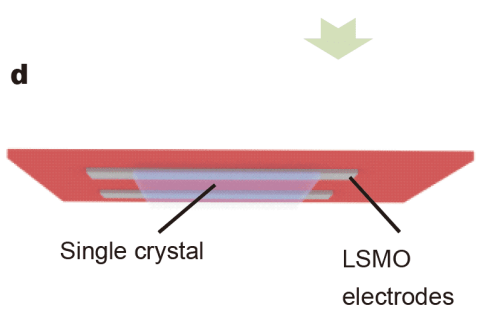

Figure 1 The procedures to fabricate the single-crystalline OSVs. (a, b) Growth of single crystals on a liquid surface. (c, d) Transfer of the single crystal to the STO substrate with patterned LSMO bottom electrodes. (e) Thermal evaporation of Au/Co top electrodes. 
strates were gently washed by deionized water and dried overnight.

It was observed that as the concentration of the solution increased from 1 to $2.5 \mathrm{mg} \mathrm{mL}^{-1}$, the color of the grown crystals changed from bright to dark green (at a fixed $100 \mu \mathrm{L}$ volume on a $300 \mathrm{~nm}-\mathrm{SiO}_{2} / \mathrm{Si}$ substrate, Fig. $2 \mathrm{a}-\mathrm{d}$ ), corresponding to an increase of the thicknesses of the crystals from 35 to $475 \mathrm{~nm}$ (Fig. S1). POM images of the crystal on the $300 \mathrm{~nm} \mathrm{SiO}_{2} / \mathrm{Si}$ substrate are shown in Fig. 2e, f and Fig. S2. The color of the whole crystal changed homogeneously from bright to dark when the substrate was rotated by $45^{\circ}$, indicating the single-crystalline nature of the checked film. A typical TEM image of a randomly selected crystal on a copper grid is shown in Fig. $2 \mathrm{~g}$ and the corresponding SAED pattern is shown in Fig. $2 \mathrm{~h}$. The SAED patterns could be indexed with previously reported lattice parameters $(a=7.565 \AA, b=$ $7.750 \AA, c=16.835 \AA, \alpha=89.15^{\circ}, \beta=92.713^{\circ}$, and $\gamma=$ $83.63^{\circ}$ ) [41]. Fig. 2 i, $j$ depict the AFM images of TIPSpentacene crystals (35 and $105 \mathrm{~nm}$ in thickness) transferred onto $\mathrm{SiO}_{2} / \mathrm{Si}$ substrates, which exhibit atomically flat surfaces with low roughness of 0.49 and $0.62 \mathrm{~nm}$, respectively. Such atomical flat surface also indicates the single-crystalline nature of the film and is favorable for device fabrication. Fig. $2 \mathrm{k}$ shows the XRD of the TIPS- pentacene single crystal. The sharp diffraction peaks indicate the high-crystalline nature of the crystal. The diffraction pattern consistently exhibits (00l) reflections, which is in accord with previous reports [42]. The primary diffraction peak (001) at $2 \theta=5.31^{\circ}$ is attributed to a $d$-spacing of $16.62 \AA$ and is equal to the $c$ axis of the TIPS-pentacene single crystal. Only (00l) diffraction peaks appear, indicating that the (001) plane of the single crystal is parallel to the substrate plane.

\section{Device fabrication}

The procedure to fabricate the OSV based on organic single crystals is sketched in Fig. 1c-e. The organic single crystals of TIPS-pentacene grown on the liquid substrate were semi-freestanding (Fig. 1a, b) and could be transferred to any solid substrates. In this study, these crystals were transferred to STO substrates with patterned LSMO bottom electrodes (Fig. 1c, d). Since the relative positions of the crystals were anchored under the assistance of the viscous glycerol substrate, it was convenient to achieve accurate transfer while maintaining crystal integrity. Top $\mathrm{Au} / \mathrm{Co}$ electrodes were prepared by thermal evaporation (Fig. 1e). It is noted that most of the reported intermediate layer materials in OSVs are either amorphous or polycrystalline thin films prepared by thermal evapora-
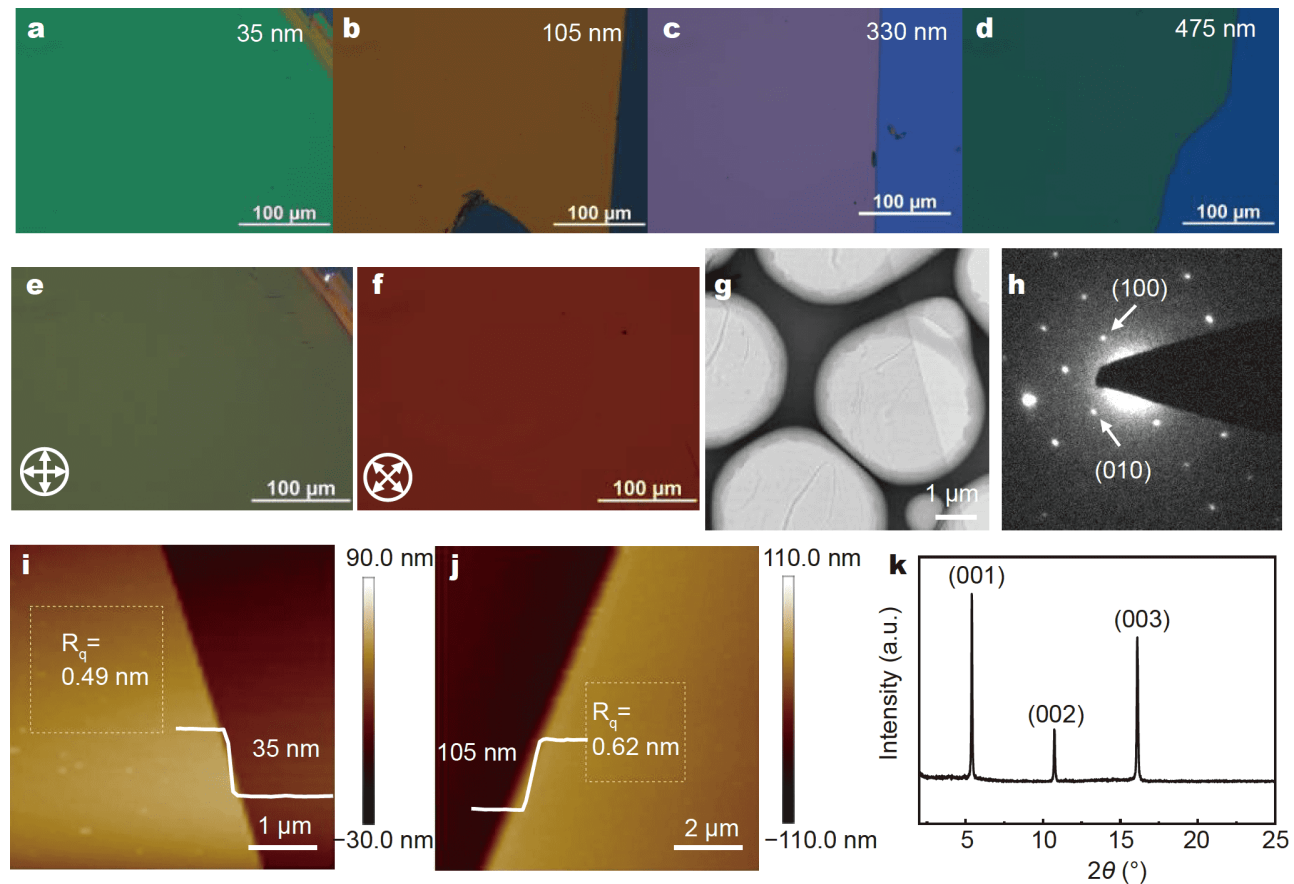

Figure 2 (a-d) OM images showing the changes of color of TIPS-pentacene single crystals as the thickness varied. (e, f) POM images of a TIPSpentacene crystal $(35 \mathrm{~nm})$ on a $\mathrm{SiO}_{2} / \mathrm{Si}$ substrate. $(\mathrm{g}, \mathrm{h})$ TEM image and the corresponding SAED pattern of a TIPS-pentacene single crystal. (i, j) AFM images of the TIPS-pentacene single crystals. (k) XRD patterns of the single crystal. 
tion or spin-coating. The use of glycerol as a liquid substrate to grow single crystals provides a reliable method for fabricating single-crystalline OSVs.

The structure of the as-prepared OSV is shown in Fig. 3a. The Fermi levels $\left(E_{\mathrm{F}}\right)$ of both the FM metals are between the lowest unoccupied molecular orbital (LUMO) and the highest occupied molecular orbital (HOMO) level of TIPS-pentacene (Fig. 3b) [43], which is beneficial for the injection and detection of spin-polarized charge carriers. The surface morphology of the LSMO electrode after patterning with dimensions of $60 \mathrm{~nm}$ (thickness) $\times 200 \mu \mathrm{m}$ (width) $\times 5 \mathrm{~mm}$ (length) was investigated by AFM, and a low surface roughness of $R_{\mathrm{q}}=$ $0.70 \mathrm{~nm}$ over an area of $10 \mu \mathrm{m} \times 10 \mu \mathrm{m}$ was observed (Fig. S3). The flat surface of the LSMO is beneficial for intimate topographical contacts between the electrodes and the crystals in the OSVs. Fig. 3c shows an OM image of a typical single-crystalline OSV device. The dimension of the single crystal is millimeter scale, which is large enough to cover the junction area $(200 \mu \mathrm{m} \times 200 \mu \mathrm{m})$ without causing current leakage. Fig. $3 \mathrm{~d}$ shows the magnetic hysteresis loops $(M-H)$ of the Co and the LSMO film measured at $30 \mathrm{~K}$. The coercive fields $\left(H_{c}\right)$ of the Co layer and LSMO film are 180 and 58 Oe, respectively. With such a large difference of $H_{c}$, it is possible to switch the relative magnetization direction of the two FM electrodes between parallel and antiparallel configurations by sweeping the external magnetic field [5].

Polycrystalline thin films of TIPS-pentacene were also prepared by vacuum thermal evaporation for comparison (Fig. S4a, b). The thicknesses are varied from 97 to $227 \mathrm{~nm}$. Compared with single crystals, polycrystalline thin films show greater roughness, $R_{\mathrm{q}}=3.34 \mathrm{~nm}$, according to AFM measurements (Fig. S4c). XRD of the polycrystalline thin film exhibits weaker and broader (00l) diffraction peaks (Fig. S4d). OFETs with a topcontact ( $\mathrm{Au}$ source and drain electrodes) bottom-gate configuration were constructed, and the typical transfer and output characteristics of the OFETs are shown in Fig. S5. The hole mobilities are 0.98 and $0.08 \mathrm{~cm}^{2} \mathrm{~V}^{-1} \mathrm{~s}^{-1}$ for single crystals and polycrystalline films, respectively.

\section{MR response}

I- $V$ measurements of the devices based on TIPS-penta-
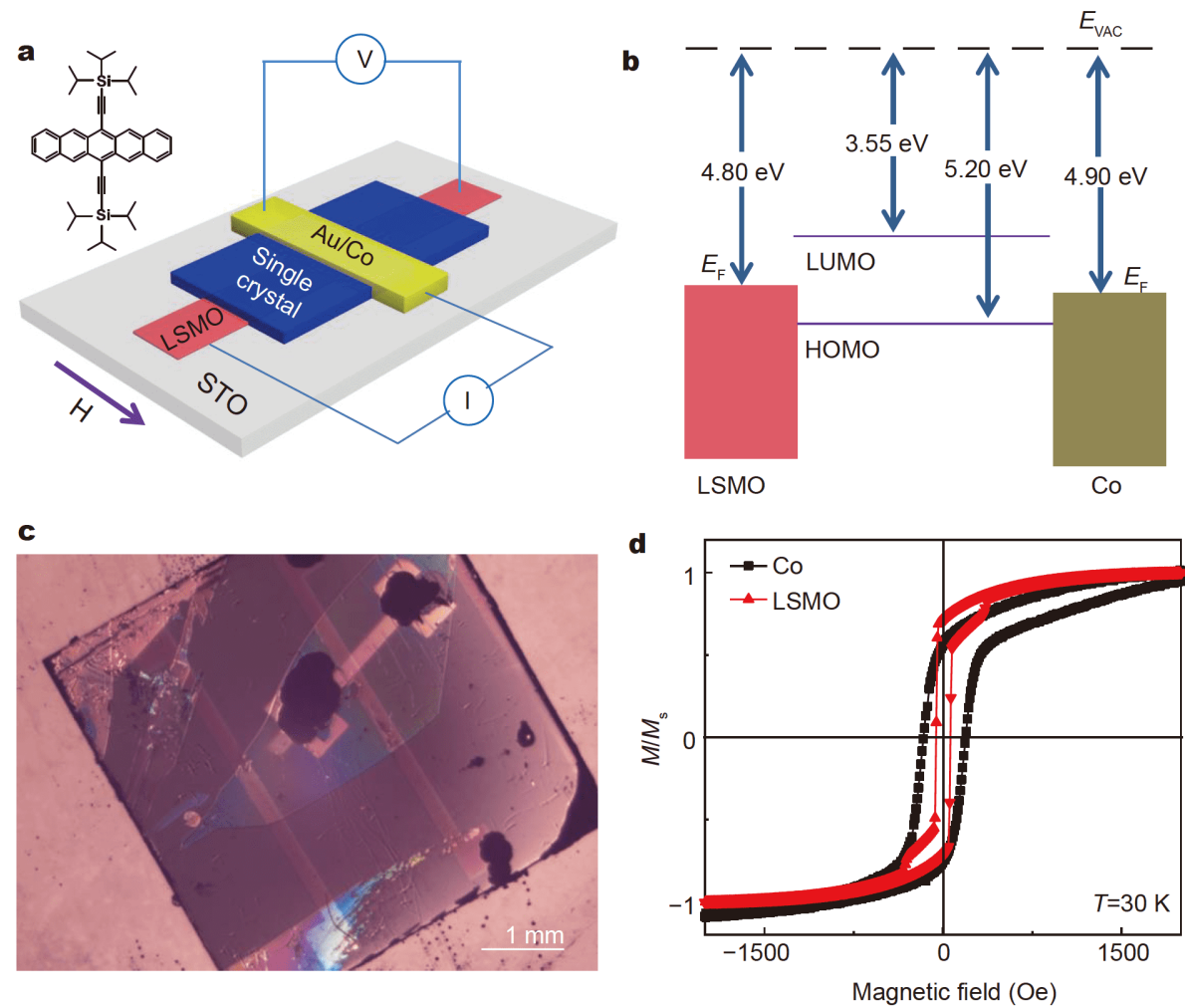

Figure 3 (a) Schematic diagram of the device with a sandwich structure of LSMO/TIPS-pentacene/Co/Au. (b) Schematic energy level diagram of the OSV device. (c) OM image of a device with a large-area TIPS-pentacene single crystal. (d) Magnetization hysteresis loops for individual electrodes of Co (black square) and LSMO (red triangle) at $T=30 \mathrm{~K}$, respectively. 
cene single crystal $(260 \mathrm{~nm})$ at different temperatures were carried out to exclude the possibility of short circuits (Fig. S6). At low $V$, a clear nonlinear $I-V$ curve can be observed. With decreasing temperature, the nonlinearity became stronger owing to the increase of device resistance at low temperatures (Fig. S6b). The resistance increased more than $778 \%$ when lowering the temperature from 300 to $5 \mathrm{~K}$ at $0.5 \mathrm{~V}$ (Fig. S6c). Judging from the $I-V$ curves, short circuit could be ruled out $[44,45]$.

Fig. 4a shows the typical MR curves based on a crystal with a thickness of $269 \mathrm{~nm}$ at $30 \mathrm{~K}$. A negative MR value as high as $17 \%$ was calculated according to the following equation [21]:

$M R=\left[R_{(\mathrm{AP})}-R_{(\mathrm{P})}\right] / R_{(\mathrm{AP})}$,

where $R_{\mathrm{AP}}$ and $R_{\mathrm{P}}$ denote the resistances of the antiparallel and parallel magnetization configurations, respectively. The square shape and rapid response at both the parallel and antiparallel states indicated that the device was well stabilized and reliable $[2,46]$. In addition, the resistance of the device was much greater than that of the LSMO itself (Fig. S7a), indicating that the resistance of the intermediate layer dominated the resistance of the device. Surprisingly, substantial MR ( $>1.5 \%)$ could still be observed in single-crystalline OSVs with TIPS-pentacene thickness as large as $457 \mathrm{~nm}$ at $30 \mathrm{~K}$ (Fig. 4b).

To investigate the origin of the MR effect in the singlecrystalline OSVs, the anisotropic MR (AMR) and tunneling anisotropic MR (TAMR) effects were evaluated. The MR effect for the full length of the LSMO electrode was measured at $30 \mathrm{~K}$ (Fig. S7a). The MR value was calculated to be approximately $+0.51 \%$. Compared with the negative and large MR signals observed in the OSV devices, the MR of the LSMO electrode was small and positive; therefore, the observed MR was not due to the AMR effect of the LSMO electrode (the AMR of Co electrode could be neglected due to the much smaller resistance compared with that of the crystal [5]). In addition, the TAMR effect, which can also cause MR signals $[5,47]$, was examined by fabricating devices with a structure of LSMO/TIPS-pentacene crystal/Au (Fig. S7b, c). No TAMR effects in the devices were identified. Judging from the above results, the MR of the OSV devices was attributed to the spin-dependent transport in the TIPS-pentacene single crystal. Inverse MR signals were observed in the crystals, which were due to the hybridization-induced filtering effects $[7,48,49]$, i.e., the "spinterface" [50]. When Co was deposited on the organic semiconductor, new hybrid electronic states formed at the interface, which collectively acted as a spin-
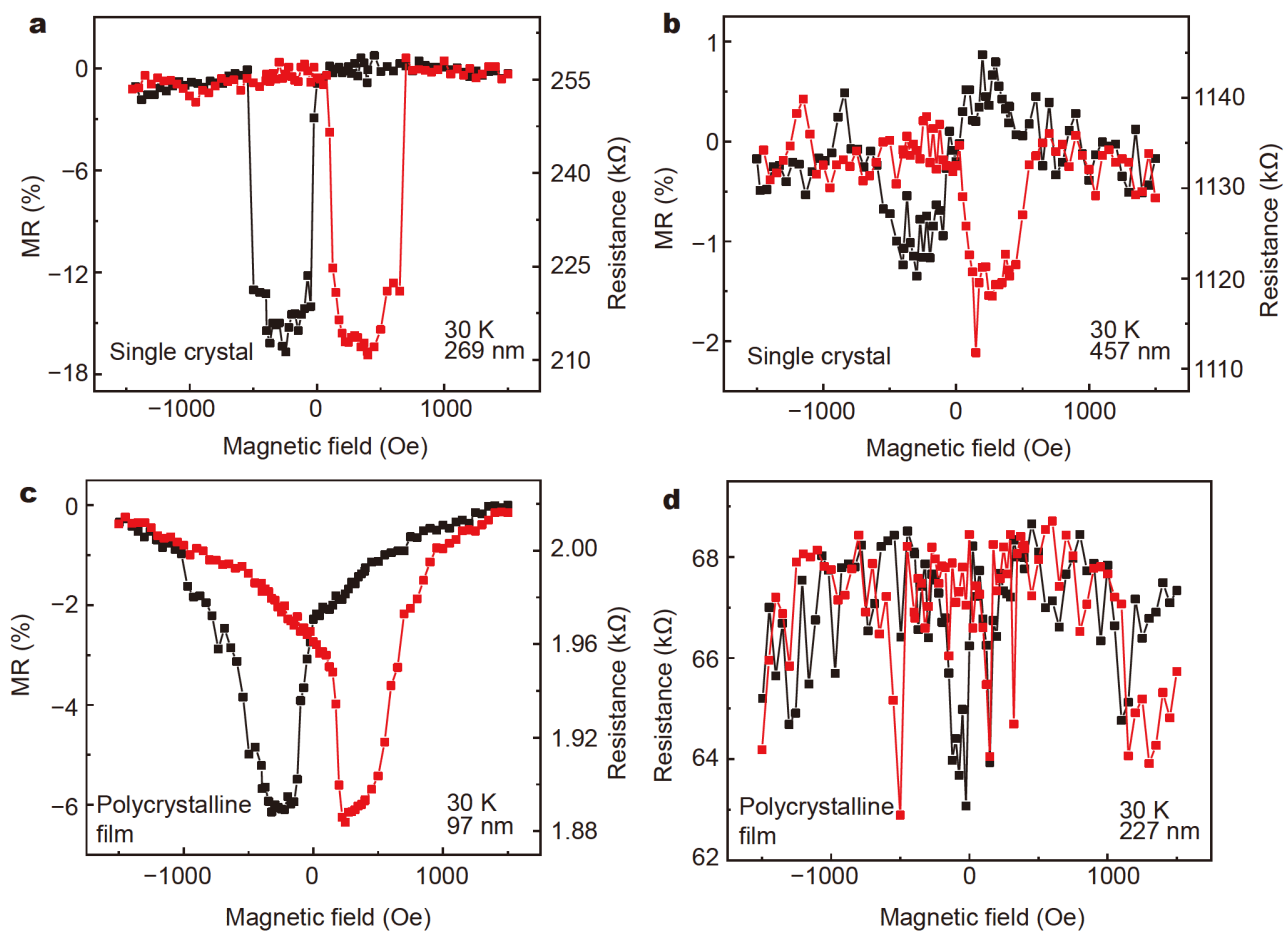

Figure 4 (a, b) MR responses of the single-crystalline OSVs at $30 \mathrm{~K}$ with TIPS-pentacene single crystals of thicknesses of 269 and $457 \mathrm{~nm}$, respectively. (c, d) MR responses of the polycrystalline thin-film OSVs at $30 \mathrm{~K}$ with thin film thicknesses of 97 and $227 \mathrm{~nm}$, respectively. 

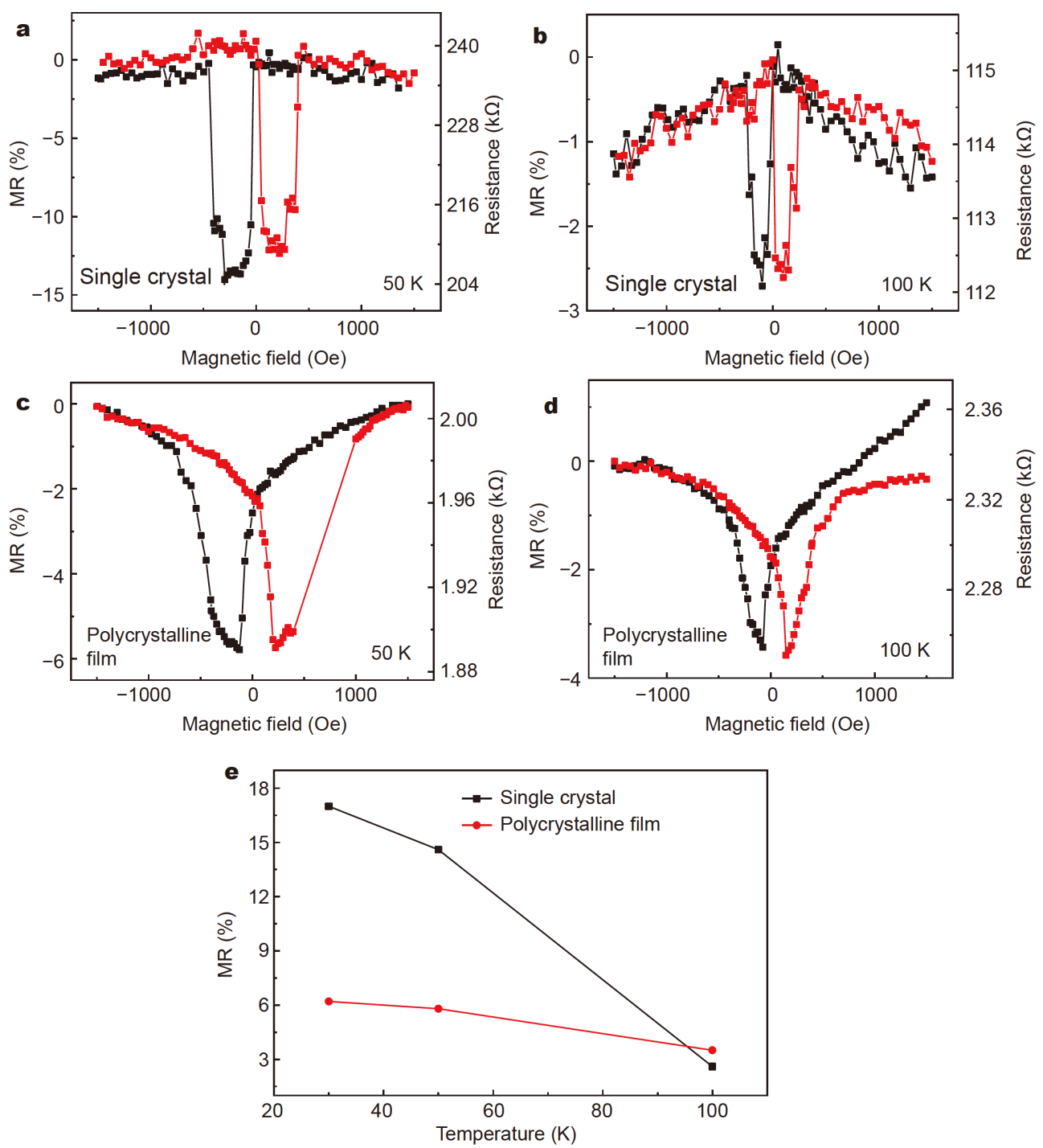

Figure 5 (a, b) MR responses of the single-crystalline OSVs with 269-nm-thick TIPS-pentacene single crystals at $T=50$ and $100 \mathrm{~K}$, respectively. (c, d) MR responses of the polycrystalline thin-film-based OSVs with a 97-nm-thick TIPS-pentacene layer at $T=50$ and $100 \mathrm{~K}$, respectively. (e) The dependence of MR ratios on the measurement temperature for single-crystalline and polycrystalline thin-film-based OSVs.

filter, leading to the inversion of the sign of the spinpolarization of the current from that of the Co electrodes and the inverse MR signals of the OSVs [50].

Fig. 4c shows a typical MR loop of an OSV device based on the polycrystalline thin film with a thickness of $97 \mathrm{~nm}$ at $30 \mathrm{~K}$. Triangular background curves were observed, indicating that either the antiparallel or parallel state was not well stabilized [46,51]. This could be caused by the rough interface between the FM electrodes and the TIPSpentacene layer, which induced inhomogeneous magnetostatic fields and thus resulted in the random precession of spin carriers [52]. It was found that the MR ratio of the polycrystalline thin film with a thickness of $97 \mathrm{~nm}$ at $30 \mathrm{~K}$ was approximately $6 \%$, which was smaller than the value of $17 \%$ for the single-crystalline OSVs with a crystal thickness of $269 \mathrm{~nm}$. According to a modified Jullière equation [21,53], MR decreases with the thickness. Therefore, the MR value of OSV based on single crystal would be much higher than that of polycrystalline film if single crystal and polycrystalline film of the same thickness were used. MR signal was undetectable when the polycrystalline thin film was of $227 \mathrm{~nm}$ thickness (Fig. 4d). In comparison, MR signal was still detectable when the thickness of the single crystal was as large as $457 \mathrm{~nm}$.

Figs $4 \mathrm{a}, \mathrm{c}$ and 5 show the temperature dependence of 
MR curves for the OSV devices based on single crystals and polycrystalline films, respectively. Between 30 and $100 \mathrm{~K}$, square MR response curves and steep changes at both the parallel and antiparallel states were observed in single-crystalline OSVs. In contrast, the MR curves showed triangular background for polycrystalline thinfilm OSVs. It was observed that the MR value monotonically decreased with increasing temperature in both devices (Fig. 5e), which could be attributed to the decrease of surface spin polarization of the LSMO electrode or the reduction of the spin diffusion length in the organic layer with increasing temperature $[2,17,44,54-57]$.

The better spin transport properties of the single crystals were attributed to their higher mobility. Due to the absence of grain boundaries and minimal defect density, the mobility of the single crystals $\left(0.98 \mathrm{~cm}^{2} \mathrm{~V}^{-1} \mathrm{~s}^{-1}\right)$ was over one order of magnitude higher than that of polycrystalline thin films $\left(0.08 \mathrm{~cm}^{2} \mathrm{~V}^{-1} \mathrm{~s}^{-1}\right)$. As a result, less spin-flip scattering was suffered during the charge and spin transport processes in single crystals $[3,30]$. In addition, compared with the larger roughness $\left(R_{\mathrm{q}}=3.34 \mathrm{~nm}\right)$ of the polycrystalline thin film, an atomically flat surface of single crystals $\left(R_{\mathrm{q}}=\right.$ $0.62 \mathrm{~nm}$ ) will further decrease scattering at the interface [58]. Therefore, long spin diffusion lengths can be expected in single-crystalline OSVs.

\section{CONCLUSIONS}

In conclusion, the first working single-crystalline vertical OSV based on TIPS-pentacene was constructed, and the MR responses were investigated. Thin and large-area organic single crystals were grown on a liquid substrate and transferred onto target substrates to fabricate singlecrystalline OSVs. An MR value as large as $17 \%$ was probed. More importantly, spin transport was still observed in a single crystal with a thickness up to $457 \mathrm{~nm}$, which was much larger than that of polycrystalline thin films. The results indicate that organic single crystals are good medium for spin transport.

Received 18 January 2021; accepted 22 March 2021; published online 28 May 2021

1 Dediu VA, Hueso LE, Bergenti I, et al. Spin routes in organic semiconductors. Nat Mater, 2009, 8: 707-716

2 Nguyen TD, Wang F, Li XG, et al. Spin diffusion in fullerene-based devices: Morphology effect. Phys Rev B, 2013, 87: 075205

3 Jang HJ, Richter CA. Organic spin-valves and beyond: Spin injection and transport in organic semiconductors and the effect of interfacial engineering. Adv Mater, 2017, 29: 1602739

4 Liu X, Zhu X, Ding S, et al. Organic spin valves and their magnetoresistance effect. Prog Chem, 2019, 31: 1199-1212
5 Zhang X, Mizukami S, Kubota T, et al. Observation of a large spindependent transport length in organic spin valves at room temperature. Nat Commun, 2013, 4: 1392

6 Li F, Li T, Chen F, et al. Spin injection and transport in organic spin-valves based on fullerene $\mathrm{C}_{60}$. Org Electron, 2014, 15: 16571663

7 Barraud C, Seneor P, Mattana R, et al. Unravelling the role of the interface for spin injection into organic semiconductors. Nat Phys, 2010, 6: 615-620

8 Sun X, Vélez S, Atxabal A, et al. A molecular spin-photovoltaic device. Science, 2017, 357: 677-680

9 Geng R, Daugherty TT, Do K, et al. A review on organic spintronic materials and devices: I. Magnetic field effect on organic light emitting diodes. J Sci-Adv Mater Devices, 2016, 1: 128-140

10 Devkota J, Geng R, Subedi RC, et al. Organic spin valves: A review. Adv Funct Mater, 2016, 26: 3881-3898

11 Dediu V, Hueso LE, Bergenti I, et al. Room-temperature spintronic effects in $\mathrm{Alq}_{3}$-based hybrid devices. Phys Rev B, 2008, 78: 115203

12 Wagemans W, Koopmans B. Spin transport and magnetoresistance in organic semiconductors. Phys Status Solidi B, 2011, 248: 10291041

13 Sun X, Bedoya-Pinto A, Mao Z, et al. Active morphology control for concomitant long distance spin transport and photoresponse in a single organic device. Adv Mater, 2016, 28: 2609-2615

14 Tsurumi J, Matsui H, Kubo T, et al. Coexistence of ultra-long spin relaxation time and coherent charge transport in organic singlecrystal semiconductors. Nat Phys, 2017, 13: 994-998

15 Bobbert PA, Wagemans W, van Oost FWA, et al. Theory for spin diffusion in disordered organic semiconductors. Phys Rev Lett, 2009, 102: 156604

16 Naber WJM, Faez S, van der Wiel WG. Organic spintronics. J Phys D-Appl Phys, 2007, 40: R205-R228

17 Sun D, Ehrenfreund E, Valy Vardeny Z. The first decade of organic spintronics research. Chem Commun, 2014, 50: 1781-1793

18 Gobbi M, Novak MA, Del Barco E. Molecular spintronics. J Appl Phys, 2019, 125: 240401

19 Ciudad D, Gobbi M, Kinane CJ, et al. Sign control of magnetoresistance through chemically engineered interfaces. Adv Mater, 2014, 26: 7561-7567

20 Yang W, Shi Q, Miao T, et al. Achieving large and nonvolatile tunable magnetoresistance in organic spin valves using electronic phase separated manganites. Nat Commun, 2019, 10: 3877

21 Xiong $\mathrm{ZH}, \mathrm{Wu} \mathrm{D}$, Valy Vardeny $\mathrm{Z}$, et al. Giant magnetoresistance in organic spin-valves. Nature, 2004, 427: 821-824

22 Gobbi M, Golmar F, Llopis R, et al. Room-temperature spin transport in $\mathrm{C}_{60}$-based spin valves. Adv Mater, 2011, 23: 1609-1613

23 Lin R, Wang F, Wohlgenannt M, et al. Organic spin-valves based on fullerene $\mathrm{C}_{60}$. Synth Met, 2011, 161: 553-557

24 Ikegami T, Kawayama I, Tonouchi M, et al. Planar-type spin valves based on low-molecular-weight organic materials with $\mathrm{La}_{0.67} \mathrm{Sr}_{0.33} \mathrm{MnO}_{3}$ electrodes. Appl Phys Lett, 2008, 92: 153304

25 Nguyen TD, Hukic-Markosian G, Wang F, et al. Isotope effect in spin response of $\pi$-conjugated polymer films and devices. Nat Mater, 2010, 9: 345-352

26 Nguyen TD, Ehrenfreund E, Vardeny ZV. Spin-polarized lightemitting diode based on an organic bipolar spin valve. Science, 2012, 337: 204-209

27 Majumdar S, Laiho R, Laukkanen P, et al. Application of regioregular polythiophene in spintronic devices: Effect of interface. Appl Phys Lett, 2006, 89: 122114 
28 Ding S, Tian Y, Li Y, et al. Inverse magnetoresistance in polymer spin valves. ACS Appl Mater Interfaces, 2017, 9: 15644-15651

29 Gershenson ME, Podzorov V, Morpurgo AF. Colloquium: Electronic transport in single-crystal organic transistors. Rev Mod Phys, 2006, 78: 973-989

30 Guo L, Qin Y, Gu X, et al. Spin transport in organic molecules. Front Chem, 2019, 7: 428

31 Yang $\mathrm{F}$, Cheng $\mathrm{S}$, Zhang $\mathrm{X}$, et al. 2D organic materials for optoelectronic applications. Adv Mater, 2018, 30: 1702415

$32 \mathrm{Li} \mathrm{R}, \mathrm{Hu} \mathrm{W}$, Liu Y, et al. Micro- and nanocrystals of organic semiconductors. Acc Chem Res, 2010, 43: 529-540

33 Naber WJM, Craciun MF, Lemmens JHJ, et al. Controlled tunnelcoupled ferromagnetic electrodes for spin injection in organic single-crystal transistors. Org Electron, 2010, 11: 743-747

34 Ding S, Tian Y, Li Y, et al. Organic single-crystal spintronics: Magnetoresistance devices with high magnetic-field sensitivity. ACS Nano, 2019, 13: 9491-9497

35 Yao J, Zhang Y, Tian X, et al. Layer-defining strategy to grow twodimensional molecular crystals on a liquid surface down to the monolayer limit. Angew Chem Int Ed, 2019, 58: 16082-16086

36 Sakamoto K, Ueno J, Bulgarevich K, et al. Anisotropic charge transport and contact resistance of 6,13-bis(triisopropylsilylethynyl) pentacene field-effect transistors fabricated by a modified flow-coating method. Appl Phys Lett, 2012, 100: 123301

37 Wang S, Zhou S, Tong Y, et al. Dielectric selection for solutionprocessed high-mobility TIPS-pentacene microwire field-effect transistors. Adv Mater Interfaces, 2019, 6: 1801984

38 Liu S, Wu JK, Fan CC, et al. Large-scale fabrication of field-effect transistors based on solution-grown organic single crystals. Sci Bull, 2015, 60: 1122-1127

39 Zhao X, Zhang B, Tang Q, et al. Conformal transistor arrays based on solution-processed organic crystals. Sci Rep, 2017, 7: 15367

40 Sakamoto K, Bulgarevich K, Miki K. Small device-to-device variation of 6,13-bis(triisopropylsilylethynyl)pentacene field-effect transistor arrays fabricated by a flow-coating method. Jpn J Appl Phys, 2014, 53: 02BE01

41 Anthony JE, Brooks JS, Eaton DL, et al. Functionalized pentacene: Improved electronic properties from control of solid-state order. J Am Chem Soc, 2001, 123: 9482-9483

42 Kim J, Jeong J, Cho HD, et al. All-solution-processed bottom-gate organic thin-film transistor with improved subthreshold behaviour using functionalized pentacene active layer. J Phys D-Appl Phys, 2009, 42: 115107

43 Wang H, Huang J, Xing S, et al. Improved mobility and lifetime of carrier for highly efficient ternary polymer solar cells based on TIPS-pentacene in PTB7:PC ${ }_{71}$ BM. Org Electron, 2016, 28: 11-19

44 Wang FJ, Yang CG, Vardeny ZV, et al. Spin response in organic spin valves based on $\mathrm{La}_{2 / 3} \mathrm{Sr}_{1 / 3} \mathrm{MnO}_{3}$ electrodes. Phys Rev B, 2007, 75: 245324

45 Li F, Li T, Chen F, et al. Excellent spin transport in spin valves based on the conjugated polymer with high carrier mobility. Sci Rep, 2015, 5: 9355

46 Ding S, Tian Y, Wang H, et al. Reliable spin valves of conjugated polymer based on mechanically transferrable top electrodes. ACS Nano, 2018, 12: 12657-12664

47 Gould C, Rüster C, Jungwirth T, et al. Tunneling anisotropic magnetoresistance: A spin-valve-like tunnel magnetoresistance using a single magnetic layer. Phys Rev Lett, 2004, 93: 117203

48 Velev JP, Dowben PA, Tsymbal EY, et al. Interface effects in spinpolarized metal/insulator layered structures. Surf Sci Rep, 2008, 63:
$400-425$

49 Caruso AN, Schulz DL, Dowben PA. Metal hybridization and electronic structure of tris(8-hydroxyquinolato)aluminum ( $\left.\mathrm{Alq}_{3}\right)$. Chem Phys Lett, 2005, 413: 321-325

50 Sanvito S. the rise of spinterface science. Nat Phys, 2010, 6: 562564

51 Vinzelberg H, Schumann J, Elefant D, et al. Low temperature tunneling magnetoresistance on $(\mathrm{La}, \mathrm{Sr}) \mathrm{MnO}_{3} / \mathrm{Co}$ junctions with organic spacer layers. J Appl Phys, 2008, 103: 093720

52 Tran TLA, Le TQ, Sanderink JGM, et al. The multistep tunneling analogue of conductivity mismatch in organic spin valves. Adv Funct Mater, 2012, 22: 1180-1189

53 Julliere M. Tunneling between ferromagnetic films. Phys Lett A, 1975, 54: 225-226

54 Yue FJ, Shi YJ, Chen BB, et al. Manipulating spin injection into organic materials through interface engineering. Appl Phys Lett, 2012, 101: 022416

55 Liang SH, Geng R, Zhang QT, et al. Large magnetoresistance at high bias voltage in double-layer organic spin valves. Org Electron, 2015, 26: 314-318

56 Kawasugi Y, Ujino T, Tada H. Room-temperature magnetoresistance in organic spin-valves based on a $\mathrm{Co}_{2} \mathrm{MnSi}$ Heusler alloy. Org Electron, 2013, 14: 3186-3189

57 Drew AJ, Hoppler J, Schulz L, et al. Direct measurement of the electronic spin diffusion length in a fully functional organic spin valve by low-energy muon spin rotation. Nat Mater, 2009, 8: 109114

58 Zhang X, Ma Q, Suzuki K, et al. Magnetoresistance effect in rubrene-based spin valves at room temperature. ACS Appl Mater Interfaces, 2015, 7: 4685-4692

Acknowledgements The authors thank Prof. Wenbo Mi (Tianjin University, China) for fruitful discussions. The authors acknowledge financial support from the National Natural Science Foundation of China (61674116, 51873148, 51633006, and 52003190), the Ministry of Science and Technology of China (2016YFA0202302) and the Natural Science Foundation of Tianjin (18JC-YBJC18400).

Author contributions Ding S, Li R and Hu W conceived the idea and directed the project. Wang Y carried out most of the experiments. Yao J promoted the method of growing single crystals. Wang X, Wu D, Jin C and Cui D prepared the LSMO electrode; Guo S fabricated the OSV devices; Yang S and Zhang L prepared the OFETs based on the polycrystalline thin films of TIPS-pentacene. Tian X performed the TEM measurements. Wang Y, Ding S and Li R wrote the paper. All authors analyzed the experimental results and contributed to the general discussion.

Conflict of interest The authors declare that they have no conflict of interest.

Supplementary information online version of the paper. 


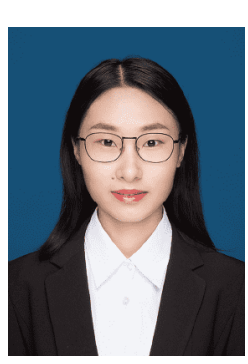

Ying Wang obtained her bachelor's degree from Qufu Normal University in 2018. She is currently a graduate student at the School of Science, Tianjin University. Her main research interests are spin injection and transport in organic semiconductors.

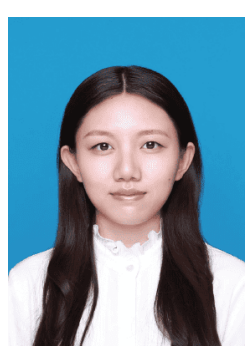

Shuaishuai Ding is a lecturer at Tianjin University. She received her $\mathrm{PhD}$ degree from the Institute of Chemistry, Chinese Academy of Sciences (CAS) in 2019, supervised by Prof. Wenping $\mathrm{Hu}$ and Prof. Daoben Zhu. Her research interest is organic spintronics.

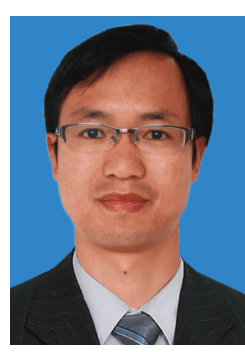

Rongjin $\mathbf{L i}$ is a professor at Tianjin University. He received his $\mathrm{PhD}$ degree from the Institute of Chemistry, CAS in 2009, supervised by Prof. Wenping Hu. His research interests include organic semiconductors, 2D molecular crystals, and organic optoelectronic devices.

\section{TIPS-pentacene单晶有机自旋阀的自旋注入与传} 输特性研究

王颖 ${ }^{1}$, 姚佳荣 ${ }^{1}, 丁$ 帅帅 ${ }^{{ }^{*}}$, 郭思宇 ${ }^{1}$, 崔大鹏 ${ }^{2}$, 王新月 ${ }^{3}$, 杨书院 ${ }^{1}$, 张利娟，田馨孜，吴镝 ${ }^{2}$, 金朝 $^{3}$, 李荣金 ${ }^{*}$, 胡文平 ${ }^{1,4}$

摘要 与非晶或多晶相比, 有机半导体单晶具有完美的晶体结构和 较少的结构缺陷, 因而具有高迁移率和低自旋散射, 使其有望在自 旋电子器件中获得长自旋驰豫时间和长自旋扩散长度. 然而, 目前 缺乏构筑单晶有机自旋阀(OSVs)的方法, 阻碍了单晶有机半导体 的自旋注入和输运特性的研究. 针对这一挑战, 本文提出了在液态 衬底表面生长大面积薄层有机单晶, 并转移至带有磁性电极的衬 底以构筑有机单晶自旋阀的策略. 我们成功探测到了TIPS-pentacene有机单晶在不同温度和不同厚度下的磁电阻响应, 获得了高 达 $17 \%$ 的磁阻. 更重要的是, 在厚达 $457 \mathrm{~nm}$ 的单晶中仍然可以观察 到自旋输运, 比多晶薄膜的自旋输运长度大得多. 据我们所知, 本研 究是第一例工作的垂直结构有机单晶自旋阀. 本研究为构建单晶 OSV提供了一种通用的方法, 为基于单晶的有机半导体本征自旋 输运特性的研究奠定了基础. 